\title{
Des systèmes productifs locaux aux systèmes locaux de compétences : une réflexion à partir du cas français
}

\author{
Régis Guillaume \\ Université de Toulouse-Le Mirail
}

\section{Introduction}

$F$ ormulant ses vœux aux forces vives de la Nation, le Président de la République annonçait avec vigueur, au début de l'année 2005, le retour d'une " grande ambition industrielle pour la France ». Devançant de quelques semaines les conclusions du rapport confié à Jean-Louis Beffa, PDG de Saint-Gobain, il prônait, à cette occasion, la mise en œuvre d'une politique offensive qui, s'appuyant sur nos champions nationaux autour desquels se grefferait un tissu dense de PME-PMI (Petite et moyennes entreprises - Petites et moyennes industries) participant de concert à l'inévitable course à l'innovation, permettrait à la France de consolider sa place au sein des grandes puissances industrielles. Relativement discrète depuis les années 1980, une politique industrielle volontariste semble renaître de ses cendres.

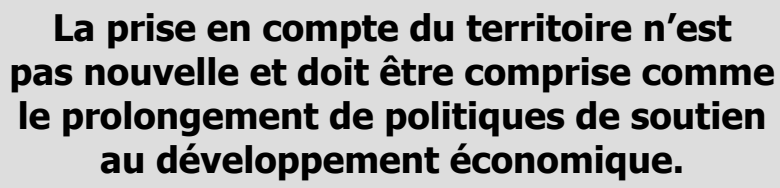

développement économique, n'est pas nouvelle et doit être comprise comme le prolongement de politiques de soutien au développement économique qui, des dispositifs pôles de conversion aux systèmes productifs locaux, accordent aux initiatives locales une place désormais prépondérante. Cependant, les objectifs qui sont fixés au dispositif «Pôle de compétitivité », l'excellence au niveau international, comme l'ampleur des moyens financiers qui lui sont attribués (une enveloppe de plus de 700 millions d'euros portée à 1,4 milliard en juillet 2005 , dont près de la moitié sur le budget propre de l'État), marquent indéniablement une inflexion en ce domaine. Bien évidemment, l'effet d'aubaine que représentent les volumes financiers adossés à cet appel d'offres décuple, en ces temps de rationalité budgétaire, les vertus pédagogiques d'une initiative dont il reste encore à vérifier l'efficacité sur le long terme. Il faut donc rester pruDans le cadre de l'appel à projets «Pôles de compétitivité » lancé par la DATAR (Délégation à l'aménagement du territoire et à l'action régionale), la qualité et le nombre de réponses portées par les régions ont surpris ses inspirateurs et amené le gouvernement à sélectionner 67 projets en lieu et place des 10 à 20 initialement prévus. Faut-il y voir l'adhésion de la « France d'en bas » à cette nouvelle priorité nationale ? Les initiatives locales se sont en effet multipliées pour parvenir à mettre en œuvre, dans des délais relativement brefs, des programmes ambitieux associant entreprises, organismes de recherche et de formation qui, selon les promoteurs de cette initiative, doivent permettre de gagner « la bataille économique grâce aux territoires $\gg$.

La prise en compte du territoire, identifié comme « vecteur» et non plus comme simple réceptacle du dent face à l'enthousiasme qui entoure cette démarche, même si elle semble marquer l'entrée dans une nouvelle phase.

Les priorités sont en effet d'une autre nature que celles qui caractérisaient les initiatives précédentes, et notamment le soutien aux 96 systèmes productifs locaux. Il s'agit désormais de « répondre » à une menace externe qui saperait les bases mêmes du tissu industriel national en soutenant les zones les plus dynamiques et performantes du territoire national plutôt que de disperser les moyens vers des sites dotés d'un tissu productif plus banal.

Un tel contexte fragilise les fondations d'un corpus théorique qui, après bien des vicissitudes et tâtonnements, est désormais intégré par les chercheurs spécialisés dans l'étude des rapports économie(s)-terri- 
toire(s). Les configurations spécifiques qui en découlent, telles que districts, technopôles et, plus récemment, systèmes productifs locaux, ne seraient pas de taille à offrir une alternative crédible à la puissance du rouleau compresseur conférée à la mondialisation.

Cet article se propose d'interroger, dans une première partie, la place dévolue au local dans l'analyse des trajectoires territoriales. La seconde est consacrée à la présentation des recherches conduites par une équipe toulousaine pluridisciplinaire qui, à travers le concept de système local de compétences, souhaite éclairer des aspects nouveaux dans la compréhension des rapports parfois ambigus qui existent entre économie(s) et territoire(s).

\section{Le risque est grand de survaloriser le rôle de la mondialisation dans les mutations en cours}

\section{Quelle place pour le « local » dans la compréhension des trajectoires territoriales?}

Dans un univers de flux, les qualités du lieu semblent quelque peu en déshérence, alors que la pertinence de postures de recherche dont l'objectif est de « découvrir » de nouvelles modalités de développement économique à partir du décryptage de ressources territoriales spécifiques s'estompe. Le rang, la taille, l'accessibilité au «réseau » sont désormais les facteurs essentiels qui conditionnent des styles de trajectoires territoriales. Pourtant, parallèlement, un consensus relativement large s'impose depuis quelques années pour décliner les principes d'une mondialisation qui entraîne à la fois des processus de standardisation, mais aussi des trajectoires territoriales singulièrement différenciées. Pour C. Manzagol le fondement de ces «particularismes » trouve son origine dans « la multiplication des interactions entre toutes les parties de la planète, son fonctionnement en système dont tous les éléments sont interdépendants, reliés par de multiples boucles de rétro-action, [et] appellent une prise en compte de l'échelle du local au mondial, à laquelle se nouent les jeux d'acteurs $\gg{ }^{1}$. Ces propos incitent à explorer l'hypothèse d'une co-occurrence entre la perception intuitive de trajectoires territoriales spécifiques et la découverte de leur sens, en les reliant à des logiques qui s'expriment à d'autres échelles. Cette piste est celle que se propose de suivre J. Lévy quand il évoque « le rôle joué par l'ancrage des lieux : il faut bien que les réseaux soient amarrés par des têtes et que les flux qui circulent sur les arcs aillent d'un lieu à l'autre. La mondialisation n'efface pas le rôle décisif des localisations; valorisant des positions anciennes, favorisant l'émergence de nouvelles. Bien au contraire, elle contribue à mettre en valeur certains lieux, ce qui à l'inverse peut alors provoquer la dévalorisation d'autres. De nouvelles singularités émergent qui résultent de relectures multiples des héritages accumulés et des influences extérieures liées à la position relative du lieu à différentes échelles $»^{2}$.

Le risque est donc grand de survaloriser le rôle de la mondialisation dans les mutations en cours, alors que, comme l'indique P. Hugon, si ce concept « permet de désigner des réalités nouvelles qui marquent profondément le monde actuel, [sa sublimation] présente le risque de gommer, d'effacer d'autres réalités tout à fait fondamentales $»^{3}$. La piste défrichée, dans le même ouvrage, par O. Dollfus, C. Grataloup et J. Lévy, est alors d'un précieux recours pour dépasser cette difficulté. Ils suggèrent de penser la mondialisation comme un processus, qui "n'invente guère, mais [qui] re-contextualise tout $\gg{ }^{4}$; en réfutant une posture qui, selon une approche fractale, consisterait à repérer à chaque niveau géographique une réplication du niveau immédiatement supérieur. La mondialisation ne saurait remettre en cause un paradigme éprouvé en géographie qui confère à chaque échelle géographique une unité de sens.

Ce choix correspond à la recommandation formulée par H. Gumuchian et al. qui revendiquent, pour « accéder à la dimension intrinsèquement territoriale de notre rapport à l'espace $»^{5}$, de ne pas négliger les approches micro, y compris pour révéler le fonctionnement des échelles plus englobantes. Elle est, selon ces auteurs, particulièrement adaptée pour décrypter les « enjeux et une lecture des pouvoirs à l'œuvre, et notamment des politiques s'exprimant dans le champ de l'action publique territoriale. Dynamiques internes à un territoire, prise en considération des évolutions globales, adaptation aux cadres changeant de l'action publique et recompositions permanentes en cours sont la résultante de stratégies portées par des acteurs qui sont multicasquettes et multiterritorialisés $»^{6}$.

Cette perspective prend un écho particulier dans le champ des rapports économie-territoire. 


\section{La tentative de construction d'un concept : les systèmes locaux de compétences}

Prolongeant les travaux engagés depuis le début 1999, et bénéficiant du soutien actif du Comité consultatif régional pour la recherche et le développement technologique (CCRRDT), un groupe de chercheurs toulousains s'est constitué autour de la thématique des systèmes productifs locaux (SPL). Elle regroupe des chercheurs appartenant à cinq laboratoires (CIEU, CERS, INRA, FRAMESPA, LEREPS ${ }^{7}$ ) qui étudient depuis de nombreuses années les systèmes productifs de la région dans des champs disciplinaires qui vont de la géographie économique à la sociologie de l'in- novation, en passant par l'économie industrielle et spatiale ou l'histoire industrielle.

Un premier programme de recherche les a conduits à caractériser le fonctionnement de principales concentrations industrielles de la région Midi-Pyrénées (figure 1). Dans cette région coexistent avec des destinées variées un district industriel spécialisé (Lavelanet), un système productif plus diversifié (Mécanic vallée), des villes moyennes en reconversion (Castres-Mazamet ou Tarbes) et une métropole régionale (Toulouse) regroupant plusieurs systèmes productifs à base technologique.

\section{Figure 1 - Les sites sélectionnés}

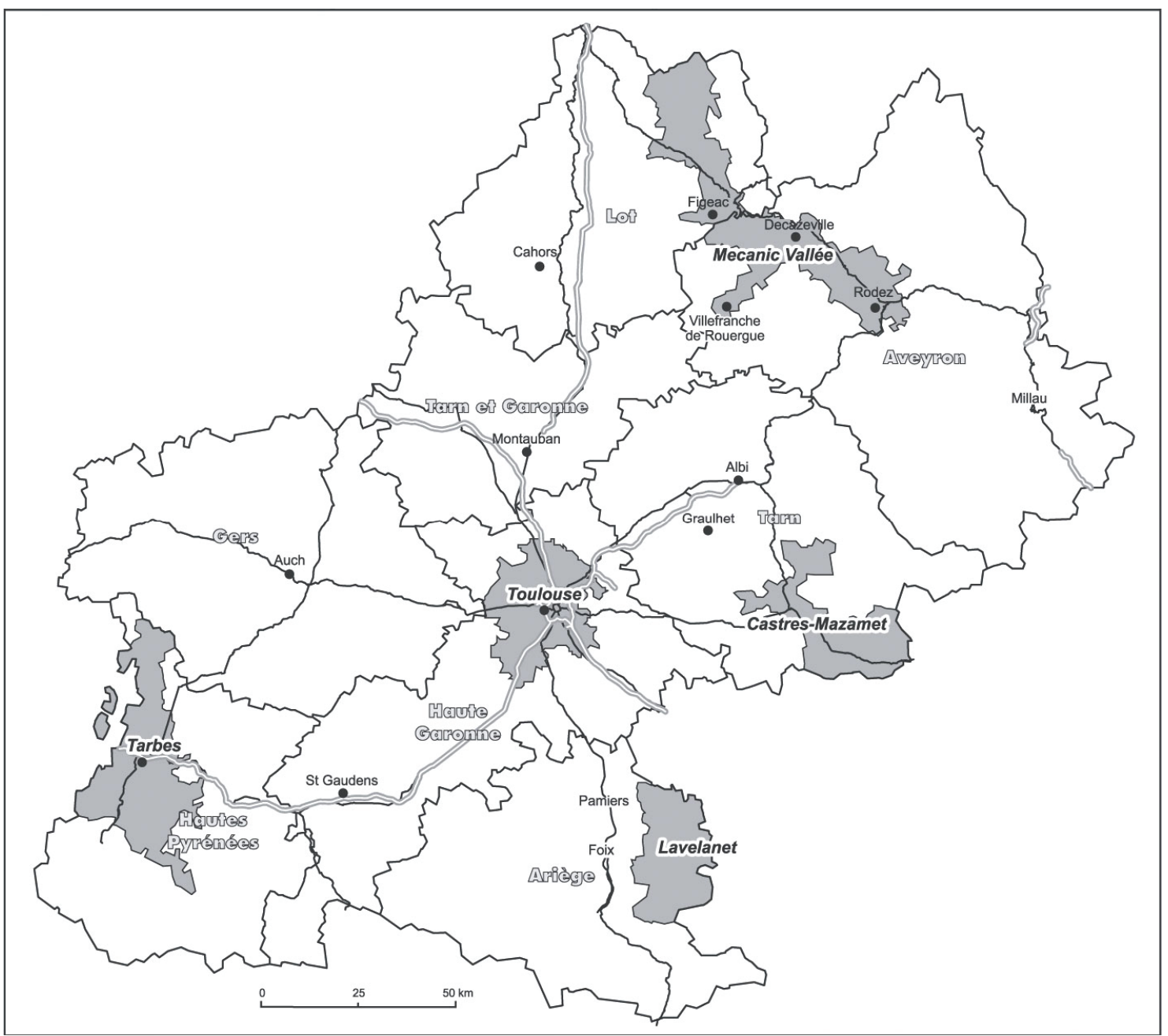

\section{Une première série de résultats}

Les investigations conduites dans la première partie du programme débouchent sur deux constats impor- tants (2000-2004). Le premier est celui de la faiblesse relative des liens intra régionaux entre les systèmes productifs étudiés. Alors même qu'était envisagée l'hypothèse de l'émergence de réseaux productifs ré- 
gionaux associant différents systèmes locaux, il s'avère que les liens régionaux sont très restreints. Par contre, les études de cas, qu'il s'agisse de systèmes productifs locaux versés dans les hautes technologies ou de ceux spécialisés dans des productions plus banales, ont permis de rendre compte d'un degré d'ouverture remarquable aussi bien au niveau national qu'international. Ils fonctionnent comme des îlots insérés dans les réseaux nationaux ou internationaux de production et d'échange.

Le second constat est celui de l'existence, au sein des sites étudiés, de systèmes de relations associant soit des firmes entre elles, soit des firmes et des organismes de recherche et de formation qui sont irréductibles à une logique de secteur d'activité traditionnellement organisé autour d'un type de produit. Dans le cas de l'agglomération toulousaine par exemple, les réseaux de coopération dessinés par les relations entre entreprises, entre laboratoires de recherche et entreprises de service sont organisés autour d'un même type de compétence technologique. Ainsi, les établissements des secteurs spatial, aéronautique et électronique forment une nébuleuse des «métiers de l'électronique et de l'informatique » au sein de laquelle s'observent de multiples relations. Mais cette nébuleuse est bien distincte de celle qui réunit les firmes du secteur des biotechnologies et de la pharmacie. En définitive, tout se passe comme si des secteurs calqués sur les découpages des départements scientifiques («sciences et technologie de l'information et de la communication ", "sciences de la vie », etc.) du CNRS (Centre national de recherche scientifique) devaient se substituer aux traditionnels secteurs d'activités organisés par produit. Un phénomène semblable est observé dans les autres sites où, là encore, les découpages selon les secteurs traditionnels résistent difficilement à l'analyse. Même si la composante R\&D est moins prégnante dans la Mécanic vallée, les évolutions les plus récentes illustrent, dans un contexte de plus en plus concurrentiel, l'affirmation de compétences et de relations entre les entreprises qui ne relèvent pas d'une seule logique sectorielle (automobile, aéronautique, machine-outil) mais de compétences transversales.

Le nord de la région Midi-Pyrénées est le théâtre d'une recomposition marquée par la prise de contrôle des plus grandes entreprises locales par des multinationales qui occupent, dans leur domaine respectif, une position de leader au niveau mondial. Ces évolu- tions traduisent un nouveau contexte spatial et organisationnel dans lequel se déroulent les activités de sous-traitance des secteurs de l'aéronautique, de l'automobile et de la machine-outil qui affectent fortement le tissu industriel de cette zone. Pour les responsables des principales firmes (locales) auxquels les avionneurs ou constructeurs automobiles confient des tâches au contenu technologique de plus en plus élaboré, ces inflexions se traduisent par une réorganisation interne des fonctions productives, par une élévation de la qualification des collectifs de travail et par un partage des "risques" liés à la mise en œuvre de tout processus innovant. Elles s'accompagnent également d'un renouvellement des relations que ces entreprises entretiennent avec les sous-traitants locaux.

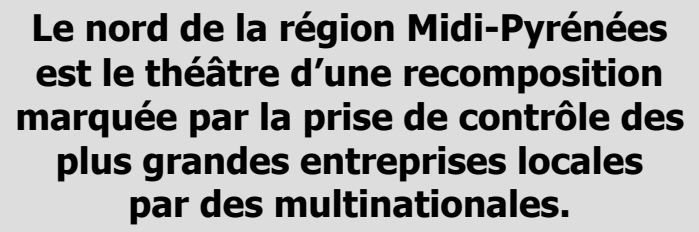

L'adoption de normes, l'exigence de qualité et de respect des délais conditionnent l'émergence d'un système relationnel associant les entreprises « leaders » et un ensemble de partenaires qui s'organise selon un registre plus réticulaire et plus flexible qu'il ne l'était durant les années 1980-1990. Les firmes principales mobilisent des ressources locales composées de deux sous-systèmes organisés autour d'un noyau d'une dizaine de sous-traitants de spécialité et d'environ 240 entreprises, de taille plus réduite, qui assurent des fonctions de sous-traitants de capacité, notamment dans les travaux d'usinage courants. Les premiers se distinguent par un niveau de compétence plus élevé et associent des activités de conception et de production dans le domaine de l'industrie aéronautique et automobile et de la machine-outil. Même s'ils n'apparaissent pas comme les principaux employeurs de la zone, les sous-traitants de spécialité complètent avantageusement le paysage industriel local en offrant une palette de compétences transversales à plusieurs secteurs d'activités. Si leur développement est lié, dans un premier temps, à une « demande » locale, ces entreprises cherchent, dans le cadre de partenariats ou de la constitution de holdings locaux, à se rapprocher des avionneurs ou des constructeurs automobiles. Ils espèrent, par ce biais, parvenir à atténuer leur dépendance par rapport aux donneurs d'ordres locaux. 


\section{Il est beaucoup plus difficile de repérer les liens entre les diverses entités présentes et, au-delà des espaces locaux, les systèmes d'échange dans lesquels elles s'inscrivent.}

La question des dispositifs d'accompagnement portés par les acteurs publics ou parapublics n'a pas été centrale dans cette première phase dont l'objectif premier visait à identifier des mutations qui relèvent prioritairement de la sphère productive. Cette posture peut surprendre alors que, depuis les lois de décentralisation de 1982 jusqu'aux réformes de la fin des années 1990, le volet développement économique des politiques territoriales s'est largement affirmé et que le renforcement de la coopération intercommunale (lois de 1992 et 1999) ou la mise en place des «Pays» (Loi d'orientation pour l'aménagement et le développement du territoire [LOADT] de 1995 et 1999) contribuent à une interpénétration plus grande entre les différentes composantes de l'action publique et de la sphère économique. Il nous a cependant semblé plus opportun de ne pas considérer cette relation comme un a priori qui expliquerait différents types de trajectoires économiques. Aussi, avons nous préféré analyser l'indéniable inflexion des politiques publiques locales comme le résultat d'un processus complexe visant certes à accompagner les mutations économiques en cours, mais qui apparait prioritairement comme un volet de stratégies plus globales promouvant la « visibilité » des territoires concernés ${ }^{8}$.

L'impact réel des multiples dispositifs et actions (aménagements de parcs d'activité, actions d'animation, soutien à des projets innovants, formations, etc.) est en effet très variable et délicat à analyser. Il ne saurait être pourtant question d'en faire l'économie à l'heure où nous souhaitons expérimenter la validité du concept de système local de compétences, pierre angulaire du programme de recherche en cours (20042008). L'objectif premier vise en effet à formuler ce concept à partir de la systématisation des méthodes permettant leur repérage et leur analyse. Il s'agit en particulier d'évaluer la nature et l'intensité des transferts de connaissances ou d'informations qui s'effectuent dans le cadre des relations mises à jour lors de la précédente phase. Le second objectif vise à apprécier l'efficience des politiques économiques locales dans la constitution de tels systèmes de relations et dans les processus d'innovation qui leur sont liés. En effet, les dispositifs censés favoriser les coopérations locales entre organisations productives et innovation se sont multipliés au cours des 20 dernières années, sans que l'on soit toujours capable d'en évaluer les effets. Or, il apparaît délicat de comprendre ces formes spatiales et leur évolution sans disposer d'une telle évaluation, même imparfaite.

\section{Les orientations méthodologiques}

La méthode choisie privilégie donc l'analyse de données qualitatives et diachroniques afin de caractériser des types de systèmes relationnels repérés dans les sites sélectionnés. Si les systèmes productifs locaux sont fondés sur des relations entre des acteurs productifs, il faut donc, pour les étudier, adopter une approche qui permette de les repérer et de les mesurer afin de re-construire ces systèmes relationnels. Or, les données habituelles de l'économie régionale ou de la géographie économique sont des données catégorielles. Il est possible de saisir de façon relativement précise des phénomènes de concentration d'entreprises, d'emplois ou d'activités dans un espace donné, mais il est beaucoup plus difficile de repérer les liens entre les diverses entités présentes et, au-delà des espaces locaux, les systèmes d'échange dans lesquels elles s'inscrivent. Lors du précédent programme, à côté des analyses traditionnelles de concentration, nécessaires dans cette phase, une part importante des efforts a été consacrée à la construction d'indicateurs relationnels. Les données disponibles sur les liens entre la recherche publique et l'industrie (les contrats CNRSentreprises en particulier) ont été mobilisées comme des données plus qualitatives, obtenues par entretiens, par questionnaires ou par sources documentaires sur les liens entre firmes (coopérations en matière de $\mathrm{R} \& \mathrm{D}$, relations client - fournisseur ou donneur d'ordres - sous-traitant). L'heure paraît venue de systématiser cette approche relationnelle en recherchant d'autres indicateurs, notamment du côté des mouvements de personnel entre les firmes et en étudiant de façon qualitative des corpus de relations pour en dégager la construction dans le temps. L'évaluation des politiques publiques est un casse-tête permanent pour les acteurs comme pour les chercheurs. Aucune méthode n'a pu, jusqu'à présent, s'imposer significativement, et les analyses conduites actuellement déconnectent, le plus souvent, l'analyse des politiques de celle de leurs effets. Aussi, la méthode choisie dans le cadre de cette investigation se veut très pragmatique. L'organisation économique des sites étudiés repose sur 
des éléments spécifiques qui ont été repérés lors de la précédente phase. Le matériau de base est donc constitué d'un corpus "d'histoires » afin d'évaluer les processus de mobilisation des ressources par les acteurs et la place que prennent, dans ces processus, les dispositifs issus de l'action publique.

\section{Conclusion/Perspectives}

Bien qu'il soit trop tôt pour évoquer les résultats d'une étude dont le terme est fixé à 2008, elle nous paraît s'inscrire pleinement dans les propos de Pecqueur ${ }^{9}$, pour qui la globalisation et la mondialisation, loin de favoriser « une marche vers une homogénéisation générale des comportements, des acteurs, des stratégies $[\ldots]$ avive les stratégies régionales voire locales $[. .$.$] qui participent à la construction de territoi-$ res, conjonction de l'espace commun abstrait construit par des groupes et d'un espace physique lorsque ce dernier contribue à l'élaboration de la ressource que fonde le "dedans" par rapport au "dehors" ».

Toutefois, si cette piste se révèle à l'évidence prometteuse, elle demande à prendre ses distances avec un $a$ priori majeur. En effet, l'appareillage conceptuel qui s'est formalisé autour des rapports économie-territoire privilégie les «régions qui gagnent». Comme le précise Moulaert ${ }^{10}$, «l'analyse occidentale du développement spatial s'est principalement concentrée sur les réussites en matière de croissance et de restructuration urbaine et régionale. L'innovation technologique, les changements professionnels et fonctionnels dans les systèmes économiques locaux ainsi que l'instauration de réseaux et de partenariats constituent le noyau terminologique du discours sur les trajectoires de développement réussies des agglomérations urbaines de premier et de second rang, sur les zones industrielles aux dynamismes de restructuration prometteurs, sur les districts industriels fondés sur le principe de l'artisanat et la haute technologie, sur les zones de services de haut niveau et sur les réseaux de régions et de villes qui gagnent ».

Or, ces configurations spécifiques sont peu nombreuses et conduisent à poser la question de la portée explicative de telles trajectoires. Est-il raisonnable d'utiliser la même grille d'analyse pour étendre les investigations au sein de territoires qui offrent un profil plus banal ? La réponse est à première vue négative puisque, à l'évidence, les fondements de telles success-stories sont, par nature, rares et difficilement reproductibles. Pourtant, l'appareillage conceptuel qui leur est lié se révèle d'une étonnante plasticité et peut être utilement mobilisé pour rendre intelligible « l'invention du territoire » que Debarbieux et Vanier ${ }^{11}$ appellent de leurs vœux. Il permet en effet de dépasser des représentations arc-boutées sur une vision de «l'espace [...] appréhendé comme une juxtaposition (principe de contiguïté) et un emboîtement de formes spatiales conçues à un niveau donné comme autant de mailles équivalentes ». C'est bien dans cette perspective que nous souhaitons expérimenter le concept de système local de compétences. L'exercice est à l'évidence ambitieux, mais il doit pouvoir tirer parti d'une démarche au caractère interdisciplinaire qui a déjà permis, par le passé, de défricher de nouvelles pistes afin de mieux saisir la nature des rapports complexes entre économie et territoire.

\section{Notes et références}

1 Manzagol, C. (2003). La mondialisation, données, mécanismes, enjeux, Paris, Armand Colin, p. 7.

2 Lévy, J. (1999). Le tournant géographique, penser l'espace pour lire le monde, Paris, Belin, p. 333.

3 Hugon, P. (1999). «L'évolution de la pensée économique et la mondialisation », dans groupe GEMDEV (dir.), Mondialisation, les mots et les choses, Karthala, p. 19-51.

4 Dollfus, O., C. Grataloup et J. Lévy (1999). « Le Monde, pluriel et singulier », dans groupe GEMDEV (dir.), Mondialisation, les mots et les choses, Karthala, p. 81-123.

5 Gumuchian, H et al. (2003). Les acteurs, ces oubliés du territoire, coll. Géographie, Anthropos, Economica, p. 6.

6 Gumuchian, H. et al., Idem, p. 46.

7 Centre interdisciplinaire d'études urbaines (CIEU), Laboratoire de recherche sur l'économie, les politiques et les systèmes sociaux (LEREPS), Centre d'études des rationalités et des savoirs (CERS) et France Méridionale et Espagne (FRAMESPA).

8 Guillaume, R. (2003). « De la hiérarchie au(x) réseau(x) : vers un renouvellement de l'analyse des villes moyennes "), dans F. Charbonneau, P. Lewis et C. Manzagol (dir.), Villes moyennes et mondialisation, Editions TRAMES, Université de Montréal, p. 164-175.

9 Pecqueur, B. (2001). « Gouvernance et régulation : un retour sur la nature du territoire », Géographie, Économie, Société, vol. $3, \mathrm{n}^{\circ}$ 2, p. 229-245.

10 Moulaert, F. (1999). «L'analyse des inégalités spatiales en Europe : contribution de l'école de la régulation », Géographie, Economie, Société, vol. 1, n 1, p. 97-133.

11 Bebarbieux, B. et M. Vanier (2002). « Les représentations à l'épreuve de la complexité territoriale : une actualité, une prospective? », dans B. Debarbieux et M. Vanier (dir.), Ces territorialités qui se dessinent, Éditions de l'Aube, p. 10. 